\title{
Prevention of postoperative visual field defect after the occipital transtentorial approach: anatomical study
}

\author{
Satoshi Matsuo, MD, PhD, ${ }^{1,2}$ Serhat Baydin, MD, ${ }^{2}$ Abuzer Güngör, MD, ${ }^{2}$ Erik H. Middlebrooks, MD, ${ }^{3}$ \\ Noritaka Komune, MD, PhD, ${ }^{4}$ Koji lihara, MD, PhD, ${ }^{5}$ and Albert L. Rhoton Jr., MD ${ }^{2}$ \\ 1Department of Neurosurgery, Kyushu Central Hospital; Departments of ${ }^{4}$ Otolaryngology Head and Neck Surgery and \\ ${ }^{5}$ Neurosurgery, Graduate School of Medical Sciences, Kyushu University, Fukuoka, Japan; ${ }^{2}$ Department of Neurosurgery, \\ University of Florida, College of Medicine, Gainesville, Florida; and ' ${ }^{3}$ Department of Radiology, University of Alabama at \\ Birmingham, School of Medicine, Birmingham, Alabama
}

OBJECTIVE A postoperative visual field defect resulting from damage to the occipital lobe during surgery is a unique complication of the occipital transtentorial approach. Though the association between patient position and this complication is well investigated, preventing the complication remains a challenge. To define the area of the occipital lobe in which retraction is least harmful, the surface anatomy of the brain, course of the optic radiations, and microsurgical anatomy of the occipital transtentorial approach were examined.

METHODS Twelve formalin-fixed cadaveric adult heads were examined with the aid of a surgical microscope and $0^{\circ}$ and $45^{\circ}$ endoscopes. The optic radiations were examined by fiber dissection and MR tractography techniques.

RESULTS The arterial and venous relationships of the lateral, medial, and inferior surfaces of the occipital lobe were defined anatomically. The full course of the optic radiations was displayed via both fiber dissection and MR tractography. Although the stems of the optic radiations as exposed by both techniques are similar, the terminations of the fibers are slightly different. The occipital transtentorial approach provides access for the removal of lesions involving the splenium, pineal gland, collicular plate, cerebellomesencephalic fissure, and anterosuperior part of the cerebellum. An angled endoscope can aid in exposing the superior medullary velum and superior cerebellar peduncles.

CONCLUSIONS Anatomical findings suggest that retracting the inferior surface of the occipital lobe may avoid direct damage and perfusion deficiency around the calcarine cortex and optic radiations near their termination. An accurate understanding of the course of the optic radiations and vascular relationships around the occipital lobe and careful retraction of the inferior surface of the occipital lobe may reduce the incidence of postoperative visual field defect.

https://thejns.org/doi/abs/10.3171/2017.4.JNS162805

KEY WORDS fiber dissection; occipital lobe; optic radiations; occipital transtentorial approach; magnetic resonance imaging tractography; striate cortex; anatomy

$\mathrm{T}$ HE occipital transtentorial approach provides exposure of both supra- and infratentorial regions including the splenium, pineal gland, collicular plate, and superior vermis and is commonly used for lesions involving these areas. ${ }^{5,18,19,23,38,42}$ One unique postoperative complication is a visual field defect that can be caused by occipital lobe damage due to brain retraction during surgery. Choosing patient positions that enable gravityassisted retraction of the occipital lobe is noted to signifi- cantly decrease, but not prevent, postoperative visual field defects. ${ }^{4,7,25,50}$ Thus, one must be cognizant of the strategic placement of retractors in the occipital lobe to minimize risks of vision-related complications. The inferior ${ }^{5,29,47}$ or medial $1^{4,14,23,41,42,50}$ surface of the occipital lobe or the occipital pole ${ }^{30,38}$ can be retracted to obtain sufficient surgical exposure for the occipital transtentorial approach (Table 1). The aim of this study was to anatomically examine the area of the occipital lobe at which retraction is least harm-

ABBREVIATIONS MCA = middle cerebral artery; PCA = posterior cerebral artery.

SUBMITTED November 14, 2016. ACCEPTED April 28, 2017.

INCLUDE WHEN CITING Published online October 20, 2017; DOI: 10.3171/2017.4.JNS162805. 
TABLE 1. Patient positions, retractor placements, and postoperative visual field defect of occipital transtentorial approach, literature review

\begin{tabular}{|c|c|c|c|}
\hline Authors \& Year & Patient Position & Retractor Placement & $\begin{array}{l}\text { Postop Visual Field Defect } \\
\text { (no. of patients/total) }\end{array}$ \\
\hline Poppen, 1966 & NA & Inferior surface & NA \\
\hline Ausman et al., 1988 & Three-quarter prone & Medial surface & None \\
\hline Stone et al., 1990 & Lateral & Medial surface & Transient: NA, permanent: $1 / 6$ \\
\hline Nazzaro et al., 1992 & Semi-sitting & Occipital pole & Transient: 12/12, permanent: 2/12 \\
\hline Little et al., 2001 & Concorde or three-quarter prone & NA & NA \\
\hline Shirane et al., 2001 & Modified Concorde & Occipital pole & None \\
\hline Yamamoto, 2001 & Three-quarter prone & Inferior surface & NA \\
\hline Tanaka \& Washiyama, 2003 & Lateral semi-prone & Medial surface & Transient: NA, permanent: $5 / 82$ \\
\hline Chi \& Lawton, 2006 & Prone \& lateral & NA & Transient: $16 / 46$, permanent: $3 / 46$ \\
\hline Moshel et al., 2009 & Three-quarter prone & Cotton balls used to maintain working space & Transient 3/22, permanent: 0/22 \\
\hline Yoshimoto et al., 2013 & $\begin{array}{l}\text { Prone w/ the head rotated toward } \\
\text { the approach side }\end{array}$ & Medial surface & Transient: 6/14, permanent: $3 / 14$ \\
\hline Hart et al., 2013 & $\begin{array}{l}\text { Prone w/ the head rotated toward } \\
\text { the approach side }\end{array}$ & Medial surface & NA \\
\hline Qiu et al., 2014 & $\begin{array}{l}\text { Prone or } 3 / 4 \text { prone } w / \text { the head } \\
\text { rotated toward the approach side }\end{array}$ & Occipital pole & Transient: 1/15, permanent: 1/15 \\
\hline McLaughlin \& Martin, 2014 & Lateral & Medial surface & Transient: 1/6, permanent: 0/6 \\
\hline Azab et al., 2014 & Three-quarter or park bench & Inferior surface & NA \\
\hline Present study & NA & Inferior surface & NA \\
\hline
\end{tabular}

$\mathrm{NA}=$ not available

ful. The vascular relationships of the occipital lobe, course of the optic radiations, and microsurgical and endoscopic anatomy of the occipital transtentorial approach were examined.

\section{Methods}

\section{Cadaveric Dissections}

The arteries and veins of 5 formalin-perfused cadaveric adult heads were injected with red and blue colored silicone and examined under an operating microscope at magnifications ranging from $\times 3$ to $\times 40$ and with $0^{\circ}$ and $45^{\circ}$ endoscopes. Bone dissection was done using a highspeed drill. The dissections followed the steps of the surgical procedures. Seven brains were formalin perfused and fixed in alcohol for fiber dissection. ${ }^{6,13}$

\section{Magnetic Resonance Imaging Tractography}

Diffusion data were provided by the Human Connectome Project (http://www.humanconnectome.org). The data were acquired on a modified 3-T Skyra system (Siemens AG) utilizing a spin-echo, echo-planar imaging sequence with multiband image acceleration. ${ }^{8,12,24,37,45}$ The specific sequence parameters used were TR $5520 \mathrm{msec}$, TE $89.5 \mathrm{msec}$, FOV $210 \times 180$, matrix $168 \times 144$, slice thickness $1.25 \mathrm{~mm}$ (voxel size $1.25 \times 1.25 \times 1.25 \mathrm{~mm}$ ), multiband factor 3, and b-values $1000 \mathrm{sec} / \mathrm{mm}^{2}$ (95 directions), $2000 \mathrm{sec} / \mathrm{mm}^{2}$ (96 directions), and $3000 \mathrm{sec} /$ $\mathrm{mm}^{2}$ (97 directions). Preprocessing of the raw diffusion data included correction of eddy current distortions, motion correction, b0 intensity normalization, susceptibility distortion correction, and gradient-nonlinearity corrections. ${ }^{1,2,12,16}$ A T1-weighted 3D magnetization-prepared rapid gradient echo (MP-RAGE) scan was registered to the diffusion data for anatomical detail. All preprocessing steps were performed with FreeSurfer (http://surfer.nmr. mgh.harvard.edu) and FSL (http://fsl.fmrib.ox.ac.uk). ${ }^{10,17}$

The preprocessed diffusion data were analyzed with DSI Studio (http://dsi-studio.labsolver.org). We estimated a voxel-wise diffusion orientation distribution function employing a generalized q-sampling imaging algorithm..$^{48}$ Streamline fiber tracking was performed by seeding regions of interest based on findings from dissection data in this study.

\section{Results}

\section{Surface Anatomy and Vascular Relationships of the Occipital Lobe}

Lateral Surface

There are no clearly defined sulci that separate the occipital lobe from the temporal and parietal lobes on the lateral surface (Fig. 1A); however, an imaginary line extending from the upper end of the parietooccipital sulcus to the preoccipital notch can be envisioned as a boundary between them.. ${ }^{11}$ The lateral surface of the occipital lobe is covered by a number of irregular sulci and gyri with considerable variability. ${ }^{32}$ The lateral occipital sulcus, the most consistent sulcus, is short and horizontal and separates the superior and inferior occipital gyri. The lateral surface of the occipital lobe is usually supplied by the branches of the middle cerebral artery (MCA) and poste- 

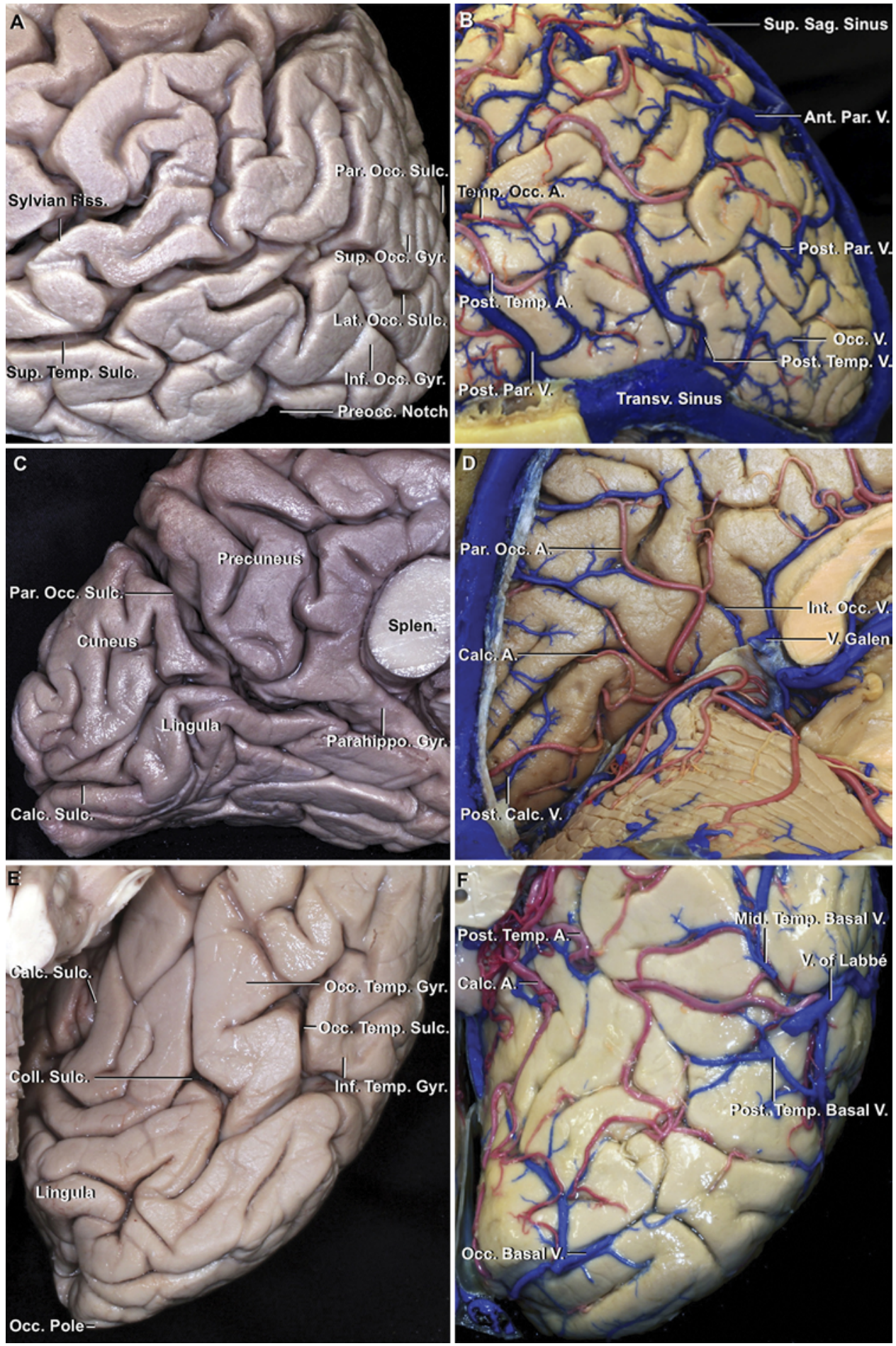

FIG. 1. Surface anatomy and vascular relationships of the posterior part of the left cerebral hemisphere. A: Lateral surface of the brain. No clearly defined sulci separate the occipital lobe from the temporal and parietal lobes on the lateral surface. An imaginary line between the end of the parietooccipital sulcus and the preoccipital notch has occasionally been used as a boundary. The lateral occipital sulcus divides the superior and inferior occipital gyri. B: Lateral surface of an injected brain. The temporooccipital and posterior temporal arteries arising from the MCA supply the lateral surface of the occipital lobe. Cortical branches from the parietooccipital, calcarine, and posterior temporal arteries arising from the PCA (not shown) send branches to supply the lateral surface of the occipital lobe. The posterior parietal vein drains the anterior part of the lateral surface of the occipital lobe. The posterior temporal vein occasionally drains the anterior part of the lateral surface of the occipital lobe. The occipital vein drains the lateral surface of the occipital lobe and is usually directed forward rather than medial or backward so that no large veins enter the superior sagittal and transverse sinuses around the torcular herophili. FIG. 1. (continued) $\rightarrow$ 
FIG. 1. C: Medial surface of the brain. The parietooccipital sulcus separates the occipital lobe from the parietal lobe. The calcarine sulcus divides this surface into the cuneus above and the lingula below. The cuneus is demarcated anteriorly by the parietooccipital sulcus, posteriorly and superiorly by the calcarine sulcus, and inferiorly by the lower border of the medial surface. Anteriorly, the lingula blends into the posterior part of the parahippocampal gyrus. D: Medial surface of an injected brain. The straight sinus has been removed. The parietooccipital artery supplies the posterior parasagittal region, cuneus, and precuneus. The calcarine artery supplies the inferior cuneus, lingual gyrus, and occipital pole. The calcarine artery sends branches to the lingual gyrus and inferior cuneus. The anterior calcarine vein, also known as the internal occipital vein, drains the anterior part of the cuneus and lingula and empties into the vein of Galen. The posterior calcarine vein drains the posterior part of the calcarine fissure and commonly empties into the veins on the lateral surface. E: Inferior surface of the brain. There are no clearly defined sulci that separate the occipital lobe from the temporal lobe on the inferior surface. The inferior surface contains the lingula and the occipitotemporal and inferior temporal gyri. The collateral sulcus separates the lingula from the occipitotemporal gyrus. The occipitotemporal sulcus separates the occipitotemporal and inferior temporal gyri. F: Inferior surface of an injected brain. The posterior temporal artery, arising from the PCA, supplies the inferior temporal and occipital surfaces. The inferior surface of the occipital lobe is drained by the occipitobasal vein, which usually courses anterolaterally to join the posterior temporobasal vein. A. = artery; Ant. = anterior; Calc. = calcarine; Coll. = collateral; Fiss. = fissure; Gyr. = gyrus; Inf. = inferior; Int. = internal; Lat. = lateral; Mid. = middle; Occ. = occipital; Occ. Basal = occipitobasal; Occ. Temp. = occipitotemporal; Par. = parietal; Par. Occ. = parietooccipital; Parahippo. = parahippocampal; Post. = posterior; Preocc. = preoccipital; Sag. = sagittal; Splen. = splenium; Sulc. = sulcus; Sup. = superior; Temp. = temporal; Temp. Basal = temporobasal; Temp. Occ. = temporooccipital; Transv. = transverse; $\mathrm{V} .=$ vein. Figure is available in color online only.

rior cerebral artery (PCA). ${ }^{34}$ The temporooccipital artery, arising from the MCA, supplies the inferior parts of the occipital gyri (Fig. 1B). ${ }^{34}$ The posterior temporal artery, also arising from the MCA, supplies the posterior extreme of the inferior temporal gyri and may supply the lateral surface of the occipital lobe ${ }^{34}$ Branches from the parietooccipital and calcarine arteries pass around the superior border of the hemisphere to reach the lateral surface (Fig. 2B). Branches from the posterior temporal arteries, arising from the PCA, pass around the lateral margin of the inferior surface to reach the lateral surface (Fig. 1F) ${ }^{34}$ The lateral surface of the anterior part of the occipital lobe is drained by the posterior parietal vein, which also drains the posterior part of the parietal lobe, and the posterior temporal vein, which also drains the posterior part of the temporal lobe (Fig. 1B). The occipital vein, which drains the lateral surface of the occipital lobe, is usually directed forward rather than medial or backward so that no large veins enter the superior sagittal and transverse sinuses around the torcular herophili (Fig. 2A). ${ }^{31}$

\section{Medial Surface}

The medial surface of the occipital lobe is separated from the parietal lobe by the parietooccipital sulcus (Fig. 1C). The parietooccipital sulcus is directed anteroinferiorly from the superior border between the cuneus and precuneus to join the anterior part of the calcarine sulcus. The parietooccipital sulcus courses approximately parallel to the line on the convexity that connects the preoccipital notch and the upper end of the parietooccipital sulcus. ${ }^{32}$ The calcarine sulcus extends forward from just above the occipital pole toward the splenium to join the parietooccipital sulcus and divides this surface into the cuneus above and the lingula below. The cuneus is demarcated anteriorly by the parietooccipital sulcus, posteriorly by the calcarine sulcus, and above by the superior border of the hemisphere. The lingula is demarcated superiorly by the calcarine sulcus and inferiorly by the lower border of the medial surface. Anteriorly, the lingula blends into the posterior part of the parahippocampal gyrus. The part of the calcarine sulcus anterior to its junction with the parietooccipital sulcus has the striate cortex on its lower lips, and the part posterior to the junction has it on both lips. ${ }^{32}$ The striate cortex, which is also known as the visual cortex, is where the optic radiations terminate (Figs. $1 \mathrm{C}$ and $3 \mathrm{C}-\mathrm{D}$ ). ${ }^{44}$

The parietooccipital and calcarine arteries, terminal branches of the PCA, supply the medial surface of the occipital lobe. The parietooccipital artery usually arises from the PCA in the ambient or quadrigeminal cistern and courses in the parietooccipital fissure to supply the posterior parasagittal region, cuneus, and precuneus (Fig. 1D) ${ }^{34}$ The calcarine artery usually arises from the PCA in the ambient or quadrigeminal cistern, but occasionally arises as a branch of the parietooccipital artery and courses in the calcarine fissure to supply the inferior cuneus, lingual gyrus, and occipital pole. ${ }^{34}$ The calcarine artery sends branches to the lingual gyrus and inferior cuneus (Figs. $1 \mathrm{D}$ and 2B). The medial surface of the occipital lobe is drained by the anterior and posterior calcarine veins (Figs. $1 \mathrm{D}$ and $2 \mathrm{~B}-\mathrm{C}$ ). The anterior calcarine vein, also known as the internal occipital vein, drains the anterior part of the cuneus and lingula and empties into the vein of Galen. The posterior calcarine vein drains the posterior part of the calcarine fissure and commonly empties into the veins on the lateral surface, which usually course forward to empty into the superior sagittal sinus (Figs. 1D and 2B). ${ }^{31}$

\section{Inferior Surface}

The inferior surface, from medial to lateral, is formed by the lower part of the lingula and the posterior parts of the occipitotemporal and inferior temporal gyri (Fig. 1E). The collateral sulcus, which extends anteriorly parallel and lateral to the calcarine sulcus from near the occipital pole, separates the lingula and occipitotemporal gyrus. The occipitotemporal sulcus, which courses parallel and lateral to the collateral sulcus, separates the occipitotemporal and inferior temporal gyri.

The posterior temporal artery, which commonly arises from the PCA in the ambient cistern, supplies the inferior temporal and occipital surfaces including the occipital pole and lingula (Fig. 1F). ${ }^{34}$ The inferior surface of the occipital lobe is drained by the occipitobasal vein, which arises from tributaries draining the inferolateral part of the lingula and adjacent parts of the occipitotemporal and 

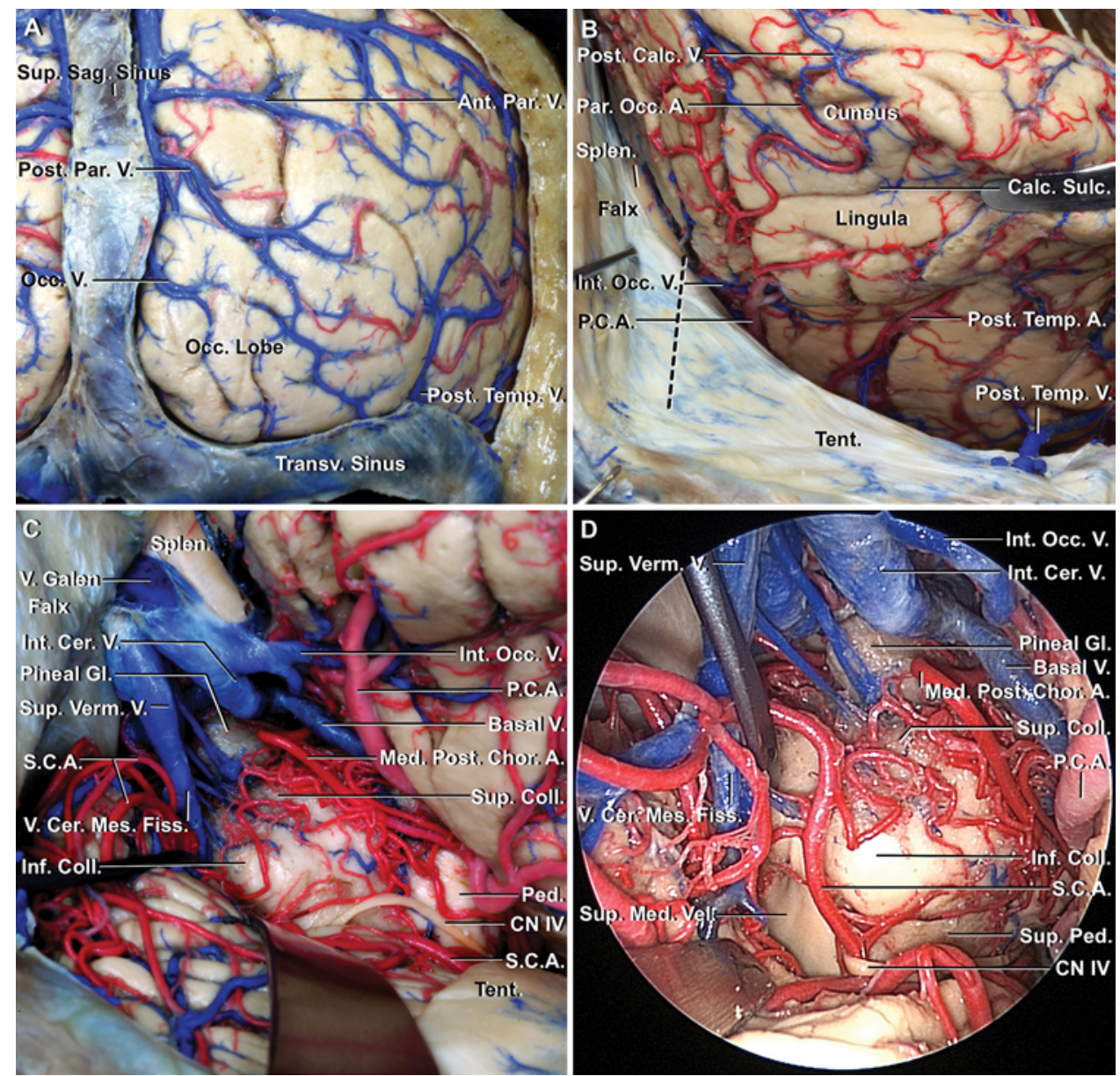

FIG. 2. Right occipital transtentorial approach. A: A right occipitoparietal craniotomy has been performed, and the dura mater covering the occipital lobe has been removed. The posterior parietal and posterior temporal veins drain the lateral surface of the anterior part of the occipital lobe. The occipital vein drains the lateral surface of the posterior part of the occipital lobe. There are no large bridging veins between the occipital lobe and superior sagittal and transverse sinuses adjacent to the torcula. B: The occipital lobe has been retracted to expose the falx and tentorium. The internal occipital vein drains the anterior part of the cuneus and lingula and empties into the vein of Galen. The posterior calcarine vein drains the posterior part of the calcarine fissure and empties into the veins on the lateral surface including the posterior parietal, posterior temporal, and occipital veins. The dashed line indicates the site of the tentorial incision. The parietooccipital, calcarine, and posterior temporal arteries send branches to supply the lateral surface of the occipital lobe. C: The tentorium has been incised lateral to the straight sinus and resected. This exposed the vein of Galen, internal occipital vein, basal vein, vein of the cerebellomesencephalic fissure, superior vermian vein, peduncle, superior and inferior colliculi, trochlear nerve, and pineal gland. The anterior part of the tentorial surface of the cerebellum is also exposed. D: A $45^{\circ}$ endoscopic view of the cerebellomesencephalic fissure exposing the superior medullary velum between the superior cerebellar peduncles. $\mathrm{A}$. = artery; Ant. = anterior; Calc. = calcarine; Cer. = cerebral; Cer. Mes. = cerebelIomesencephalic; Chor. = choroidal; $\mathrm{CN}=$ cranial nerve; Coll. = colliculus; Fiss. = fissure; $\mathrm{Gl}$. = gland; Inf. = inferior; Int. = internal; Med. = medial, medullary; Occ. = occipital; P.C.A. = posterior cerebral artery; Par. = parietal; Par. Occ. = parietooccipital; Ped. = peduncle; Post. = posterior; S.C.A. = superior cerebellar artery; Sag. = sagittal; Splen. = splenium; Sulc. = sulcus; Sup. = superior; Temp. = temporal; Tent. = tentorium; Transv. = transverse; $V_{.}=$vein; Vel. = velum; Verm. = vermian. Figure is available in color online only.

inferior temporal gyri, and usually courses anterolaterally to join the posterior temporobasal vein..$^{31}$ Occasionally, the veins draining the inferior surface of the temporal and occipital lobes empty into the medial part of the tentorial sinus near the transverse sinus..$^{22}$

\section{Optic Radiations}

The optic radiation fibers, formed by axons from the neurons of the lateral geniculate body and pulvinar and terminating in the primary visual cortex, are found on the lower lip of the calcarine sulcus anterior to the junction with the parietooccipital sulcus, the upper and lower lips of the calcarine sulcus posterior to the junction with the parietooccipital sulcus, and in part of the occipital pole (Fig. 3). ${ }^{44}$ The pulvinar is the prominent posterior part of the thalamus, and the lateral geniculate body is located anterolateral to it (Fig. 3C and G). ${ }^{32}$ After leaving the lateral geniculate body and pulvinar, the optic radiations pass inferior and posterior to the lenticular nucleus to form the sublenticular and retrolenticular parts of the internal cap- 

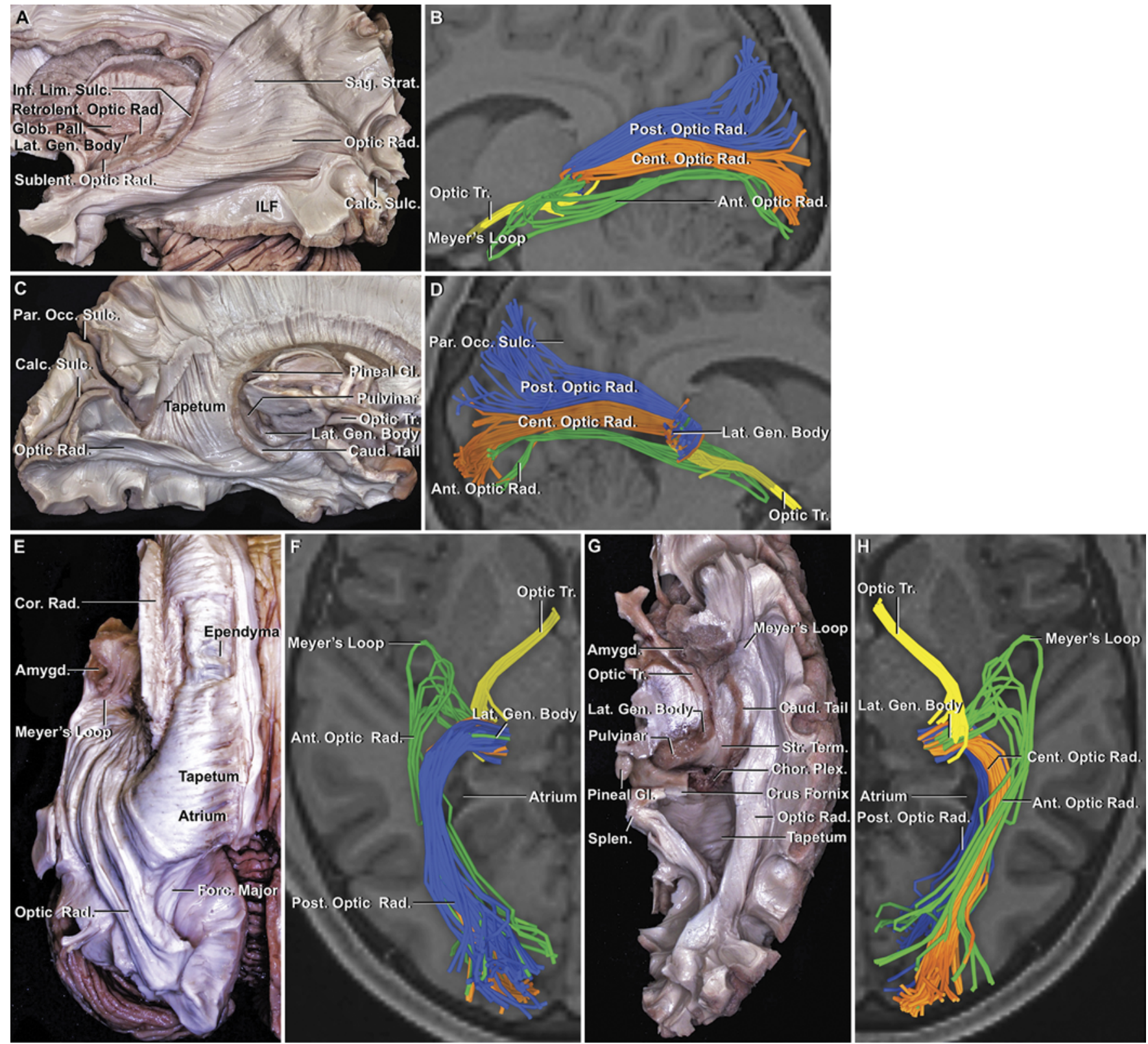

FIG. 3. Fiber dissection and MR tractography of the left optic radiations. Lateral view of the optic radiations (A). The insular cortex, extreme capsule, claustrum, ventral and dorsal parts of the external capsule, putamen, inferior frontooccipital fasciculus, anterior commissure, and part of the sagittal stratum have been removed to expose the optic radiations, which arise in the lateral geniculate body and course posterior and inferior to the lentiform nuclei to reach the calcarine cortex. Lateral view of MR tractography (B) of the optic radiations showing the optic tract (yellow) and the anterior (green), central (orange), and posterior (blue) parts of the optic radiations. After the anterior part of the optic radiations projects anteriorly, the fibers turn backward to form Meyer's loop. Medial view of the optic radiations (C). The right cerebral hemisphere, left brainstem, corpus callosum, and medial cortex of the left hemisphere have been removed. The ependyma of the left lateral ventricle has been removed to expose the tapetum fibers. The optic radiations are separated from the lateral ventricle and atrium by the tapetal fibers. The pulvinar is the prominent posterior part of the thalamus, and the lateral geniculate body is located anterolateral to it. Medial view of the MR tractography (D) shown in panel B. Superior view of the optic radiations $(E)$. The cerebral cortex and white matter above the optic radiations and tapetum have been removed. Part of the tapetal fibers covering the body of the lateral ventricle has been removed to expose the ependyma. Superior view of the MR tractography $(F)$ shown in panel B. Note that the optic radiations end near the medial surface of the occipital pole, potentially the site of greatest retraction in the occipital transtentorial approach. Inferior view of the optic radiations $(\mathbf{G})$. The cerebral cortex of the temporal base and the ependyma covering the temporal horn and atrium have been removed to expose the optic radiations coursing in the roof and lateral wall of the temporal horn. The optic fibers pass above the stria terminalis and the tail of the caudate nucleus and course lateral to the atrium and occipital horn to reach the calcarine cortex. Inferior view of the MR tractography $(\mathbf{H})$ shown in panel B. Amygd. = amygdala; Ant. = anterior; Calc. = calcarine; Caud. = caudate; Cent. = central; Chor. Plex. = choroid plexus; Cor. = corona; Forc. = forceps; Gen. = geniculate; Gl. = gland; Glob. Pall. = globus pallidus; ILF = inferior longitudinal fasciculus; Inf. = inferior; Lat. = lateral; Lim. = limiting; Par. Occ. = parietooccipital; Post. = posterior; Rad. = radiata, radiations; Retrolent. = retrolenticular; Sag. = sagittal; Splen. = splenium; Str. = stria; Strat. = stratum; Sublent. = sublenticular; Sulc. $=$ sulcus; Term. $=$ terminalis; $\operatorname{Tr} .=$ tract. Figure is available in color online only. 

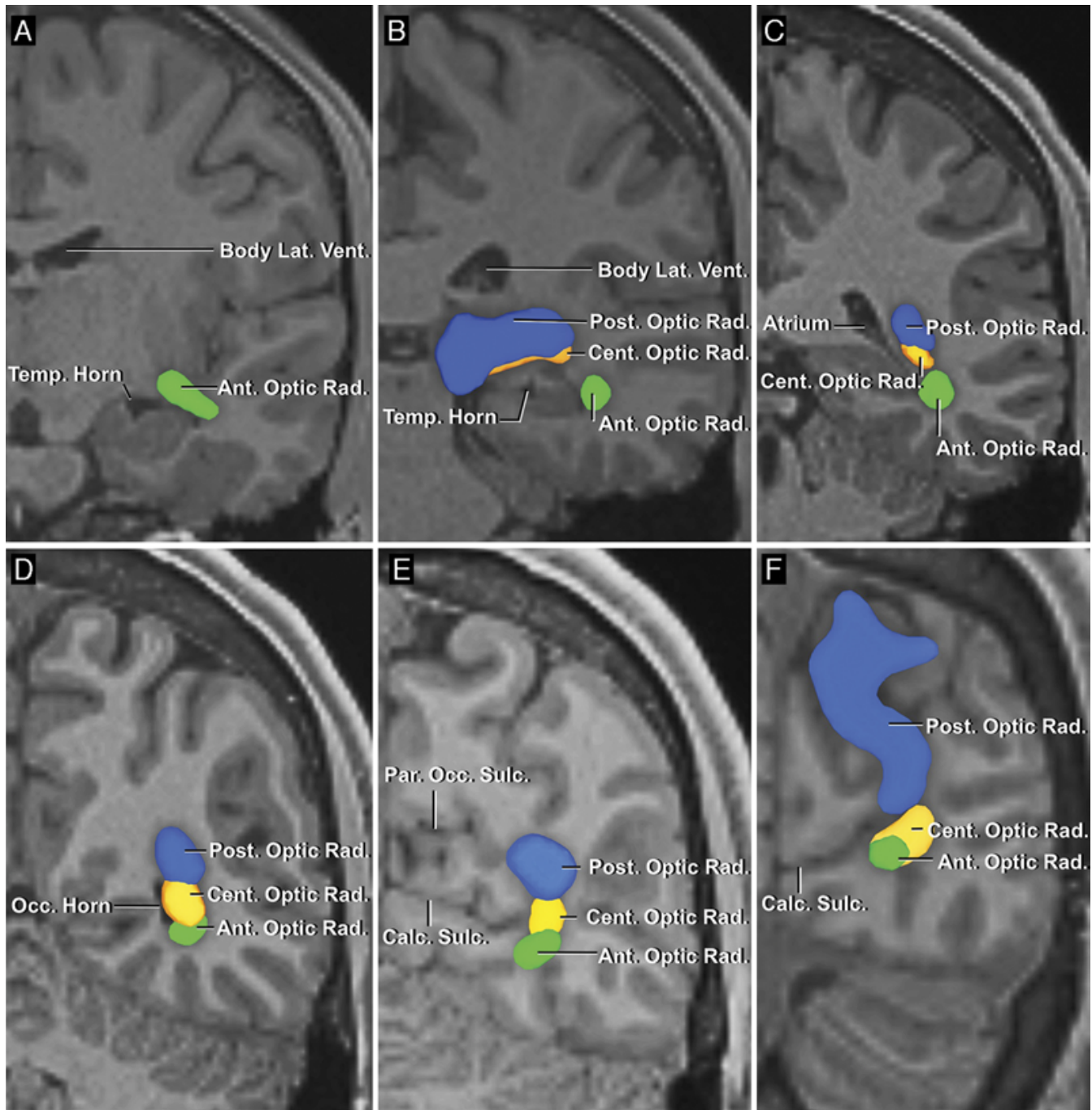

FIG. 4. Coronal views of the MR tractography shown in Fig. 3B at the level of the tip of the temporal horn (A), lateral geniculate body (B), atrium (C), occipital horn (D), junction of the parietooccipital and calcarine sulci (E), and midline between the junction and occipital pole $(\mathbf{F})$. The anterior part of the optic radiations courses in an anterolateral direction after its origin, reaches as far anteriorly as the tip of the temporal horn, then turns backward. This part covers the roof and lateral wall of the temporal horn and inferior surface of the atrium and terminates at the lower lip of the calcarine sulcus. The central part of the optic radiations initially projects laterally from its origin and then turns backward. This part covers the roof of the temporal horn and lateral wall of the atrium and occipital horn to reach the occipital pole. The posterior part of the optic radiations courses directly backward after its origin and along the lateral wall of the atrium and occipital horn to reach the upper lip of the calcarine sulcus. Ant. = anterior; Calc. = calcarine; Cent. = central; Lat. = lateral; Occ. = occipital; Par. Occ. $=$ parietooccipital; Post. = posterior; Rad. $=$ radiata, radiations; Sulc. = sulcus; Temp. = temporal; Vent. = ventricular. Figure is available in color online only.

sule, respectively (Fig. 3A). The fibers pass above the stria terminalis and tail of the caudate nucleus, cover the roof of the temporal horn and lateral wall of the atrium and occipital horn of the lateral ventricle, and reach the calcarine cortex (Fig. 3A, C, E, and G). The inferior frontooccipital fasciculus and sagittal stratum cover, and the tapetal fibers lie deep to, the optic radiation fibers (Fig. 3A, C, and E) ${ }^{46}$ The optic fibers consist of anterior, central, and posterior parts, defined by the direction the fibers projecting from their origin. ${ }^{28,46}$ It is difficult to clearly differentiate these three parts with the fiber dissection technique (Fig. 3A, $\mathrm{C}, \mathrm{E}$, and $\mathrm{G}$ ), whereas MR tractography can show each part from origin to termination (Figs. 3B, D, F, and $\mathrm{H}$ and 4). The anterior band of optic radiations courses in an anterolateral direction after its origin, usually reaching anteriorly as far as the tip of the temporal horn, then the fibers turn backward and form a loop (Meyer's loop; Fig. $3 \mathrm{~B}, \mathrm{~F}$, and $\mathrm{H}$ ). This part covers the roof and lateral wall of the temporal horn and inferior surface of the atrium of the lateral ventricle, terminating at the lower lip of the calcarine sulcus (Figs. 3B, D, F, and H and 4). The central part of the optic radiations initially projects laterally from the lateral geniculate body and pulvinar, and then turns backward (Fig. 3B, D, F, and H). This central part covers the roof of the temporal horn and lateral wall of the atrium and occipital horn, reaching the occipital pole 
(Figs. 3B, D, F, and $\mathrm{H}$ and 4) ${ }^{28}$ The posterior part of the optic radiations courses directly backward after arising from the lateral geniculate body and pulvinar, and courses along the lateral wall of the atrium and occipital horn to reach the upper lip of the calcarine sulcus. ${ }^{28}$ The anterior, central, and posterior parts of the optic radiations course along the lateral wall of the lateral ventricle, atrium, and occipital horn in this order from inferior to superior (Fig. $4 C$ and D).

\section{Microsurgical Anatomy of the Occipital Transtentorial Approach}

The occipital transtentorial approach provides access to and is commonly used for lesions of the ipsilateral splenium above the vein of Galen, pineal gland, superior and inferior colliculi, and superior surface of the cerebellum (Fig. 2). 5,18,19,23,38,42 After occipitoparietal craniotomy and dural opening, the occipital lobe is retracted away from the falx and tentorium without sacrificing bridging veins. Fortunately, there are commonly no bridging veins from the occipital lobe entering the posterior part of the superior sagittal or transverse sinuses (Fig. 2A). ${ }^{31,33}$ This provides access to the ipsilateral splenium above the vein of Galen and to part of the quadrigeminal cistern (Fig. 2B). Lateral reflection or wedge-shaped resection of the tentorium increases the exposure. Dissecting the arachnoid over the quadrigeminal cistern exposes the pineal gland, superior and inferior colliculi, trochlear nerve, and vein of Galen and its tributaries, including the internal occipital, internal cerebral, basal, and superior vermian veins, and the vein of the cerebellomesencephalic fissure (Fig. 2C). Although a steep superior cerebellar surface may limit microsurgical access to the cerebellomesencephalic fissure, an angled endoscope may overcome this obstruction and aid in the exposure of the superior cerebellar peduncle and superior medullary velum (Fig. 2D).

\section{Discussion}

The advantage of the occipital transtentorial approach is that it provides vertically wide operative views of ipsilateral midline neurovascular structures from the splenium to the cerebellomesencephalic fissure and of the anterior part of the tentorial surface of the cerebellum (Figs. 3 and 4). ${ }^{5,18,23,38}$ The deficit unique to this approach is a postoperative homonymous hemianopia that is usually transient but occasionally permanent (Table 1 ). The frequency of the postoperative visual field defect varies in different studies. Shirane et al. ${ }^{38}$ and Ausman et al. ${ }^{4}$ reported no postoperative visual field defect after the occipital transtentorial approach, whereas Nazzaro et al. ${ }^{26}$ reported that all patients had this visual field defect after such an approach. This discrepancy may be attributable to transient visual field defects with recovery common within a couple of months and, occasionally, in less than 10 days. $7,25,26,50$

Although the occipital transtentorial approach has traditionally been performed with the patient in the sitting position, the approach can also be performed with the patient in the prone, Concorde, lateral, or three-quarter prone position (Table 1). Placing the patient in a lateral or threequarter prone position with the operative hemisphere tilted downward seems to be safer for the occipital lobe because these positions require less retraction than the sitting, Concorde, and prone positions. In fact, the incidence of postoperative visual field defect is significantly lower in the lateral position than in the prone position, though it does still occur. ${ }^{7,25,50}$ Regardless of the position used, the retractors should always be carefully placed in the area of the occipital lobe.

Though the course of the optic radiations has been addressed in many fiber dissection studies, ${ }^{20,28,35,36}$ none has focused on the optic radiations on the medial side of the occipital lobe, which are usually retracted for the occipital transtentorial approach. ${ }^{42}$ In the present study, we showed the location of the optic radiations in relation to the superior, inferior, lateral, and medial surfaces by using both fiber dissection and MR tractography techniques. The optic radiations terminate in the primary visual cortex, which is mainly located in the upper and lower lips of the calcarine sulcus and partially in the occipital pole, as shown in fiber dissection and MR tractography (Figs. 3 and 4). ${ }^{44}$ The optic radiations are formed by approximately 5 million axons that course in multiple directions, especially near their termination, to reach the visual cortex ${ }^{36}$ This can prevent either MRI or fiber dissection from being a precise histological technique, especially near the termination of the fibers. The fiber dissection technique is limited because of the complex relationships of the fiber tracts such that exposing one fiber tract often destroys another. ${ }^{43}$ These disadvantages can be overcome by using MR tractography, allowing the visualization of fibers from any direction and helping form a 3D understanding of fiber tract anatomy. Traditional methods of MR tractography, namely diffusion tensor imaging (DTI), suffer from serious limitations, particularly related to identification of fiber crossing and multiple fiber orientations within a single voxel. ${ }^{9}$ Newer techniques such as diffusion spectrum imaging (DSI) or generalized q-sampling imaging (GQI) offer superior fiber tracking results by generating an orientation distribution function (ODF) analyzing multiple potential fiber directions ${ }^{48}$ However, the available selection of tracking parameters still has the potential to introduce "false fiber" tracking. ${ }^{48}$ Combining the fiber dissection technique with more sophisticated measures of diffusion MR tractography, as in the present study, compensates for the potential disadvantages of each technique.

Brain retraction can cause hemorrhage and contusion not only in the cortex, but also extending into the white matter underneath the retractor. ${ }^{49}$ For the occipital transtentorial approach, either the inferior or the medial surface of the occipital lobe or the occipital pole can be retracted (Table 1). Considering the termination locations of the optic radiations, retracting the medial surface of the occipital lobe or the occipital pole could cause postoperative visual field defect. ${ }^{36,44}$ The central part of the visual field, including the foveal part, is located in the striate cortex at the occipital pole, whereas the peripheral visual field is located in the anterior part of the calcarine cortex. ${ }^{36,44}$ Retracting the occipital pole can cause central visual field defect, resulting in reading difficulties. ${ }^{27}$ This can decrease visionrelated quality of life; therefore, retracting the occipital pole should be avoided if possible. ${ }^{27}$ Retracting the inferior 
surface of the occipital lobe away from the falx and tentorium is likely to be less harmful to the optic radiations and calcarine cortex. ${ }^{5,47}$

Brain retraction can cause brain injury directly or as a result of decreased cerebral perfusion. ${ }^{3}$ Given that most postoperative visual field defects are temporary, perfusion deficiency of the occipital lobe may play an important role in the pathophysiology of the complication..$^{50}$ When retracting the occipital lobe, it is necessary to understand the vascular relationships of the occipital lobe. Shulman ${ }^{39}$ found that the mean small arterial and venous pressures in the subarachnoid space were 63 and $19 \mathrm{~mm} \mathrm{Hg}$, respectively. The average initial retraction pressure of the experienced neurosurgeon was $26.6 \mathrm{~mm} \mathrm{Hg}$ (range 8-56 mm $\mathrm{Hg}$ ), and the pressure decreased to approximately $30 \%$ of the initial pressure 30 minutes later. ${ }^{15}$ It has been noted that veins seem to be more susceptible to damage from retraction than the arteries. ${ }^{15,39}$ Venous collapse causes a disruption of venous drainage, which can result in temporary venous congestion and brain dysfunction. The internal occipital and posterior calcarine veins, which drain the calcarine cortex, course along the medial surface of the occipital lobe, indicating that retracting the medial surface may also cause postoperative visual field defect due to venous congestion in the medial surface of the occipital lobe.

Damaging the extrastriate visual cortex surrounding the calcarine cortex may also cause visual field defect. ${ }^{40}$ Gentle retraction is important even when retracting the inferior surface of the occipital lobe. ${ }^{40}$ The occipitobasal vein draining the inferior surface of the occipital lobe usually joins the posterior temporobasal vein and drains into the lateral tentorial sinus near the transverse-sigmoid sinus junction so that the lateral part of the occipital lobe is anchored at this point. ${ }^{5,31}$ When retracting the inferior surface of the occipital lobe away from the falx and tentorium, care should be taken to avoid damaging these veins. Moreover, the use of multiple retractors, evacuation of the cerebrospinal fluid, and head elevation are helpful techniques for decreasing the intraoperative cerebral damage caused by brain retraction..$^{15}$ Intermittent brain retraction is also helpful to avoid brain damage. ${ }^{49}$

\section{Conclusions}

The occipital transtentorial approach is used to access the splenium, pineal gland, collicular plate, cerebellomesencephalic fissure, and the anterosuperior part of the cerebellum. An angled endoscope aids in exposing the cerebellomesencephalic fissure, superior medullary velum, and superior cerebellar peduncle. Anatomical findings suggest that retracting the inferior surface of the occipital lobe away from the falx and tentorium may be the safest way to avoid direct damage and perfusion insufficiency around the calcarine cortex and optic radiations near their termination. Though further clinical studies are needed, selecting a patient position that requires less brain retraction combined with careful retraction of the inferior surface of the occipital lobe, if needed, can reduce the incidence of postoperative visual field defect after the occipital transtentorial approach.

\section{Acknowledgments}

The MR tractography data were provided in part by the Human Connectome Project, the Washington University-University of Minnesota Consortium (Principal Investigators: David Van Essen and Kamil Ugurbil; Grant No. 1U54MH091657) funded by the 16 NIH institutes and centers that support the NIH Blueprint for Neuroscience Research, and the McDonnell Center for Systems Neuroscience at Washington University. This work was supported by the University of Florida Foundation.

We thank Robin Barry, MA, for her assistance in the preparation of the illustrations, and Jessica Striley, BS, for her editorial assistance.

\section{References}

1. Andersson JLR, Skare S, Ashburner J: How to correct susceptibility distortions in spin-echo echo-planar images: application to diffusion tensor imaging. Neuroimage 20:870-888, 2003

2. Andersson JLR, Xu J, Yacoub E, Auerbach E, Moeller S, Ugurbil K: A comprehensive Gaussian process framework for correcting distortions and movements in diffusion images. Proc Int Soc Mag Reson Med 20:2426, 2012 (Abstract)

3. Andrews RJ, Bringas JR: A review of brain retraction and recommendations for minimizing intraoperative brain injury. Neurosurgery 33:1052-1064, 1993

4. Ausman JI, Malik GM, Dujovny M, Mann R: Three-quarter prone approach to the pineal-tentorial region. Surg Neurol 29:298-306, 1988

5. Azab WA, Nasim K, Salaheddin W: An overview of the current surgical options for pineal region tumors. Surg Neurol Int 5:39, 2014

6. Baydin S, Yagmurlu K, Tanriover N, Güngör A, Rhoton AL Jr: Microsurgical and fiber tract anatomy of the nucleus accumbens. Neurosurgery 12:ONS269-ONS288, 2016

7. Chi JH, Lawton MT: Posterior interhemispheric approach: surgical technique, application to vascular lesions, and benefits of gravity retraction. Neurosurgery 59 (1 Suppl 1):ONS41-ONS49, 2006

8. Feinberg DA, Moeller S, Smith SM, Auerbach E, Ramanna S, Gunther M, et al: Multiplexed echo planar imaging for subsecond whole brain FMRI and fast diffusion imaging. PLoS One 5:e15710, 2010 (Erratum in PLoS One 6:10.1371/annotation/d9496d01-8c5d-4d24-8287-94449ada5064)

9. Fernández-Miranda JC, Rhoton AL Jr, Alvarez-Linera J, Kakizawa Y, Choi C, de Oliveira EP: Three-dimensional microsurgical and tractographic anatomy of the white matter of the human brain. Neurosurgery 62 (6 Suppl 3):989-1028, 2008

10. Fischl B: FreeSurfer. Neuroimage 62:774-781, 2012

11. Flores LP: Occipital lobe morphological anatomy: anatomical and surgical aspects. Arq Neuropsiquiatr 60 (3-A):566571,2002

12. Glasser MF, Sotiropoulos SN, Wilson JA, Coalson TS, Fischl $\mathrm{B}$, Andersson JL, et al: The minimal preprocessing pipelines for the Human Connectome Project. Neuroimage 80:105124,2013

13. Güngör A, Baydin S, Middlebrooks EH, Tanriover N, Isler C, Rhoton AL Jr: The white matter tracts of the cerebrum in ventricular surgery and hydrocephalus. J Neurosurg 126:945-971, 2017

14. Hart MG, Santarius T, Kirollos RW: How I do it-pineal surgery: supracerebellar infratentorial versus occipital transtentorial. Acta Neurochir (Wien) 155:463-467, 2013

15. Hongo K, Kobayashi S, Yokoh A, Sugita K: Monitoring retraction pressure on the brain. An experimental and clinical study. J Neurosurg 66:270-275, 1987

16. Jenkinson M, Bannister P, Brady M, Smith S: Improved optimization for the robust and accurate linear registration and 
motion correction of brain images. Neuroimage 17:825-841, 2002

17. Jenkinson M, Beckmann CF, Behrens TE, Woolrich MW, Smith SM: FSL. Neuroimage 62:782-790, 2012

18. Kawashima M, Rhoton AL Jr, Matsushima T: Comparison of posterior approaches to the posterior incisural space: microsurgical anatomy and proposal of a new method, the occipital bi-transtentorial/falcine approach. Neurosurgery 62 (6 Suppl 3):1136-1149, 2008

19. Konovalov AN, Pitskhelauri DI: Principles of treatment of the pineal region tumors. Surg Neurol 59:250-268, 2003

20. Kucukyuruk B, Yagmurlu K, Tanriover N, Uzan M, Rhoton AL Jr: Microsurgical anatomy of the white matter tracts in hemispherotomy. Neurosurgery 10 (Suppl 2):305-324, 2014

21. Little KM, Friedman AH, Fukushima T: Surgical approaches to pineal region tumors. J Neurooncol 54:287-299, 2001

22. Matsushima T, Suzuki SO, Fukui M, Rhoton AL Jr, de Oliveira E, Ono M: Microsurgical anatomy of the tentorial sinuses. J Neurosurg 71:923-928, 1989

23. McLaughlin N, Martin NA: The occipital interhemispheric transtentorial approach for superior vermian, superomedian cerebellar, and tectal arteriovenous malformations: advantages, limitations, and alternatives. World Neurosurg 82:409-416, 2014

24. Moeller S, Yacoub E, Olman CA, Auerbach E, Strupp J, Harel N, et al: Multiband multislice GE-EPI at 7 tesla, with 16-fold acceleration using partial parallel imaging with application to high spatial and temporal whole-brain fMRI. Magn Reson Med 63:1144-1153, 2010

25. Moshel YA, Parker EC, Kelly PJ: Occipital transtentorial approach to the precentral cerebellar fissure and posterior incisural space. Neurosurgery 65:554-564, 2009

26. Nazzaro JM, Shults WT, Neuwelt EA: Neuro-ophthalmological function of patients with pineal region tumors approached transtentorially in the semisitting position. J Neurosurg 76:746-751, 1992

27. Papageorgiou E, Hardiess G, Schaeffel F, Wiethoelter H, Karnath $\mathrm{HO}$, Mallot $\mathrm{H}$, et al: Assessment of vision-related quality of life in patients with homonymous visual field defects. Graefes Arch Clin Exp Ophthalmol 245:1749-1758, 2007

28. Párraga RG, Ribas GC, Welling LC, Alves RV, de Oliveira E: Microsurgical anatomy of the optic radiation and related fibers in 3-dimensional images. Neurosurgery 71 (1 Suppl Operative):160-172, 2012

29. Poppen JL: The right occipital approach to a pinealoma. J Neurosurg 25:706-710, 1966

30. Qiu B, Wang Y, Ou S, Guo Z, Wang Y: The unilateral occipital transtentorial approach for pineal region meningiomas: a report of 15 cases. Int J Neurosci 124:741-747, 2014

31. Rhoton AL Jr: The cerebral veins. Neurosurgery 51 (4 Suppl):S159-S205, 2002

32. Rhoton AL Jr: The cerebrum. Neurosurgery 51 (4 Suppl):S1-S51, 2002

33. Rhoton AL Jr: The lateral and third ventricles. Neurosurgery 51 (4 Suppl):S207-S271, 2002

34. Rhoton AL Jr: The supratentorial arteries. Neurosurgery 51 (4 Suppl):S53-S120, 2002

35. Rubino PA, Rhoton AL Jr, Tong X, de Oliveira E: Three-dimensional relationships of the optic radiation. Neurosurgery 57 (4 Suppl):219-227, 2005

36. Schiefer U, Hart W: Functional anatomy of the human visual pathway, in Schiefer U, Wilhelm H, Hart W (eds): Clinical Neuro-Ophthalmology. Berlin: Springer, 2007, pp 19-28

37. Setsompop K, Gagoski BA, Polimeni JR, Witzel T, Wedeen VJ, Wald LL: Blipped-controlled aliasing in parallel imaging for simultaneous multislice echo planar imaging with reduced g-factor penalty. Magn Reson Med 67:1210-1224, 2012
38. Shirane R, Kumabe T, Yoshida Y, Su CC, Jokura H, Umezawa K, et al: Surgical treatment of posterior fossa tumors via the occipital transtentorial approach: evaluation of operative safety and results in 14 patients with anterosuperior cerebellar tumors. J Neurosurg 94:927-935, 2001

39. Shulman K: Small artery and vein pressure in the subarachnoid space of the dog. J Surg Res 5:56-61, 1965

40. Slotnick SD, Moo LR: Retinotopic mapping reveals extrastriate cortical basis of homonymous quadrantanopia. Neuroreport 14:1209-1213, 2003

41. Stone JL, Cybulski GR, Crowell RM, Moody RA: The lateral position--dependant occipital approach--to pineal and medial occipitoparietal lesions. Technical note. Acta Neurochir (Wien) 102:133-136, 1990

42. Tanaka R, Washiyama K: Occipital transtentorial approach to pineal region tumors. Oper Tech Neurosurg 6:215-221, 2003

43. Türe U, Yaşargil MG, Friedman AH, Al-Mefty O: Fiber dissection technique: lateral aspect of the brain. Neurosurgery 47:417-427, 2000

44. Wichmann W, Müller-Forell W: Anatomy of the visual system. Eur J Radiol 49:8-30, 2004

45. Xu J, Moeller S, Strupp J, Auerbach E, Feinberg DA, Ugurbil K, et al: Highly accelerated whole brain imaging using aligned-blipped-controlled-aliasing multiband EPI. Proc Int Soc Mag Reson Med 20:2306, 2012 (Abstract)

46. Yagmurlu K, Vlasak AL, Rhoton AL Jr: Three-dimensional topographic fiber tract anatomy of the cerebrum. Neurosurgery 11 (Suppl 2):274-305, 2015

47. Yamamoto I: Pineal region tumor: surgical anatomy and approach. J Neurooncol 54:263-275, 2001

48. Yeh FC, Wedeen VJ, Tseng WY: Generalized q-sampling imaging. IEEE Trans Med Imaging 29:1626-1635, 2010

49. Yokoh A, Sugita K, Kobayashi S: Intermittent versus continuous brain retraction. An experimental study. J Neurosurg 58:918-923, 1983

50. Yoshimoto K, Araki Y, Amano T, Matsumoto K, Nakamizo A, Sasaki T: Clinical features and pathophysiological mechanism of the hemianoptic complication after the occipital transtentorial approach. Clin Neurol Neurosurg 115:12501256,2013

\section{Disclosures}

The authors report no conflict of interest concerning the materials or methods used in this study or the findings specified in this paper.

\section{Author Contributions}

Conception and design: all authors. Acquisition of data: Matsuo, Baydin, Güngör, Middlebrooks, Komune, Iihara. Analysis and interpretation of data: Matsuo. Drafting the article: Matsuo, Baydin, Güngör, Middlebrooks, Komune, Rhoton. Critically revising the article: Matsuo, Rhoton. Reviewed submitted version of manuscript: Matsuo, Baydin, Güngör, Middlebrooks, Komune, Iihara. Approved the final version of the manuscript on behalf of all authors: Matsuo. Administrative/technical/material support: Rhoton. Study supervision: Rhoton.

\section{Correspondence}

Satoshi Matsuo, Department of Neurosurgery, Kyushu Central Hospital, 3-23-1, Shiobaru, Minami-ku, Fukuoka 815-8588, Japan. email: smatsuo1979@gmail.com. 